\title{
Protective and Promising Myogenic Effect of Two Thymoquinone Formulations In Relation To the Hamster Buccal Pouch-Induced Carcinogenic Model
}

\author{
Magda MA Hassan ${ }^{1}$, DMSc, GhadaA Abdel-Latif ${ }^{2} \mathrm{PhD}$, \\ Wafaa H El-Hossary ${ }^{2} \mathrm{PhD}$. \\ ${ }^{1,2}$ Professor of Oral Pathology, Faculty of Dentistry, Suez Canal University, Ismailia, Egypt
}

\begin{abstract}
Introduction and Aim of the study: In this study, the induced dysplasia model of hamster buccal pouch with $D M B A(H B P / D M B A)$ was used to study the effect of thymoquinone (TQ), and different concentrations of thymoquinone loaded on liposomes (NTQ) formulation, as chemo-protective-/-therapeutic agents.

Materials and Method: The study was carried out on (170) male Syrian hamsters, divided into 7 groups. TQ and different concentrations of $N T Q$, were i.p. injected daily at $0.1 \mathrm{mg} / \mathrm{kg}$ body weight for $T Q$ and $0.1,0.05$, 0.025 and $0.01 \mathrm{mg} / \mathrm{kg}$ for $N T Q$, respectively, two weeks before DMBA. Then were continued with DMBA painting 3/w/6 weeks and left untreated for 2 weeks. Untreated and DMBA only-treated animals for six weeks, served as negative and positive controls, respectively. The excised pouches were processed for $H \& E$ and Masson's trichrome stains, as well as IHC staining for TNF- $\alpha, N f-\kappa B$, TGF- $\beta 1$, and caspase 3.

Results: Topical application of DMBA produced significant shortening of the painted pouches and different grades of epithelial dysplasia up to CIS and squamous carcinoma (end of the experiment). TQ and NTQ groups (0.1, and 0.05 $\mathrm{mg} / \mathrm{kg}$ ) resulted in mild/moderate epithelial dysplasia, while NTQ $(0.025$ and $0.01 \mathrm{mg} / \mathrm{kg})$ groups severe epithelial dysplasia. Pouches of all combined groups showed variable degrees of pouches' elongation, through promyogenic effect.IHC results revealed clear expressions in favor of regressing the carcinogenic process, and expressions in favor of muscle regeneration.Masson's trichrome stain: showed a transition from fibrous tissue to muscle fiber differentiation after 2 weeks of combined DMBA and TQ treatments.

Conclusion: TQ and higher concentrations of $N T Q$ had a promising chemo-protective and -therapeutic effect in the carcinogenesis process, as well as a pro-myogenic activity even with continuous chronic irritating inflammatory and mutagenic stimulus.
\end{abstract}

Key Words: Thymoquinone, Nanothymoquinone, pro-myogenic activity, phyto-chemo-protection, phyto-chemotherapeutic, HBP/DMBA model.

\section{Introduction}

Cancer prevention especially with phytochemicals is considered a golden medal for fighting cancer. The HBP/DMBA model for cancer induction was of great help to understand the biologic interactions of cancer prevention, progression, and treatment $(1,2)$. Thymoquinone (TQ), was found to prevent or treat many malignancies (both in vivo and in vitro) (3). Its main activity was due to its anti-inflammatory effect through inactivation of NF-KB, which upon activation is translocated to the nucleus, bind to DNA in turn, activating about 500 genes (4).Most of them are related to inflammatory cytokines, angiogenic factors, proliferative stimulating agents, anti-apoptotic molecules, as well as proteins involved in the metastatic process(5).

Sethi et al (2008) found that TQ suppressed TNF-induced NF-KBactivation on a time- and dosedependent manner, with suppressing some inflammatory agents and carcinogens upon NF-KB activation $(3,4$, 6). Of the known mediators involved in the carcinogenesis process are TNF- $\alpha$, TGF- $\beta$, and caspase- 3 . TNF- $\alpha$ is a main activator of NF-KB(7), that is produced by many cells as inflammatory cells(8). TGF-ßactivity wasrelated toTNF-ainmany conditions, with conflicting reports about their interactions (9).

Nanotechnology added much to the battle against cancer for diagnosis, or treatment(10). Materials in nano size are effective in drug delivery, used in lower doses, in different ways of applications, and more biocompatible with reduced toxicity to normal cells (11-12). Toxicity of many agents were commonly evaluated through measuring AST, ALT (liver enzymes), and creatinine serum levels (kidney toxicity) $(13,14)$. TQ and NTQ, in a comparable study were found to be safe and nontoxic (15). Furthermore, TQ in different nanoformulations were studiedin vivo and in vitro, against many malignancies, and proved to be more effective $(16,17)$.

The HBP/DMBA model was found to result in significant shortening of the painted pouches (from about $5 \mathrm{~cm}$ to about $2 \mathrm{~cm}$ )(18), followed by development of squamous cell carcinoma (in 8-12 weeks) (19). The HBP is formed of four layers, a thinkeratinized stratified squamous epithelial surface, covering thin collagenous lamina propria, striated muscle layer, loose connective tissue, fat tissue, and salivary glands. After necrosis, healing takes 
place without muscle regeneration $(15,18)$. Of interest, a study in our laboratory was carried out on the effect of different NTQ concentrations as chemotherapeutic agents on that model,reported a significant elongation of the painted pouches to about $4 \mathrm{~cm}$. Histologically, the four layers were of normal appearance in particular a thicker well defined striated muscle layer, with only mild epithelial dysplasia (18).Mature muscle regeneration is affected by a balance between pro-and anti-inflammatory factors that determine fibrosis or repair by muscle fibers. The sequence is necrosis, regeneration (by stimulating stem cells to proliferate and differentiate), remodeling and finally maturation/functional repair (20).Failure of this process ends with muscle fibrosis of injured muscles, through TGF- $\beta 1$ through activating the fibrotic process(21).In support of this effect is that inhibition of TGF- $\beta$ signaling prevents fibrosis and improves regeneration in mdx mutants \{ (a mouse model for Duchenne muscular dystrophy (DMD) \}.The management of these conditions is mainly by corticosteroids or immunosuppressants with their known side effects $(22,23)$.

After muscle injury TNF- $\alpha$ is highly expressed in injured myofibers, inflammatory cells, endothelial cells, fibroblasts and mast cells(24).

The anticarcinogenic effects of TQ is activation of various apoptotic pathways, with special reference to its advantages ofspecific effect against cancer cells than normal cells(25).TQ was reported to induce apoptosis viaactivation of caspase-3, in a cell-line model (26), which is essential in fragmentation of DNA (an irreversible stage in apoptosis)(27).

Caspase-3, a key factor of the apoptotic pathway of damaged/ mutated cells, as well as in muscle regeneration $(28,29)$. A myogenic effect of caspase-3 was reported through skeletal muscle differentiation by caspase-3 activity, in both animal and cell culture models (28).

The present study was carried out to look for the chemo-protecting and -therapeutic effects of different NTQ concentrations vs.freeTQ in the HBP/DMBA model.

\section{Materials and Methods}

This work had been down before activation of the Research Ethics Committee, Faculty of Dentistry, Suez Canal University, (which was due at Jan. $3^{\text {rd }} 2017$ ).

Chemicals: DMBA $\{7,12$-dimethylbenz (a)-anthracene) $\}$, thymoquinone, and heavy mineral oil (USP) \{Sigma Aldrich Comp, Saint Louis, MO, USA\}.

Propylene glycol :( Propane-1.2-diol), \{ADWIC, El-Nasr Pharmaceutical Chemicals Co. Egypt $\}$.

Nanothymoquinone: was kindly prepared and submitted by Nano-tech Comp., 6th October, Egypt, following Odeh et al (2012) (17) with modification. It was supplied in $0.1,0.05,0.025$, and $0.01 \mathrm{mg} / \mathrm{kg}$ body weight, concentrations. They were given to the experimental groups as TQ and left untreated for 2 weeks follow up.

DMBA $(0.5 \%)$ was painted by camel hair brush \#4, to the left pouches, 3/wk/6wks.

TQ $(0.1 \mathrm{mg} / \mathrm{kg}$ body weight) was i.p. injected daily, for $2 \mathrm{wks}$, followed by combined DMBA and TQ $3 /$ wk/6wks.

IHC kits:TGFb-1 \{US Biological Life Sciences. (Rabbit anti-human TGFb-1)\}, TNF-alpha \{Bioss, USA\}, andNFkB/p65 \{Thermo-Scientific, USA\}, Caspase-3(Gene Tex.Inc). DAB-technique was used for all IHC stains,following the manufacturer's instructions.

\section{Animals and treatment protocol (Table I):}

The study was carried out on 170 male Syrian hamsters (Mesocrietus auratus), weighing 100-120 grams, from "The Holding Company for Biological Products and Vaccines (VACSERA), Helwan, Cairo, Egypt". They were housed 5 per cage and provided water and food adlibitum. The animals were housed in a controlled temperature and humidity, in the Animal House, Faculty of Dentistry, Suez Canal University, Egypt. Sacrificing:Five animals from each group were sacrificed, every two weeks, except the DMBA-only group where sacrificing started after two weeks offirst painting.

The animals were sacrificed by a lethal dose of ether inhalation and both pouches from all animals were surgically excised, fixed and embedded in paraffin blocks for H\&E and Masson's trichrome stains, as well as IHC staining.

This work was mainly a descriptive study to define the level of expression in each tissue, and reported as mild, moderate or intense.

\section{Results}

Nano-results: The prepared NTQ was spherical with a size range of 3-25nm.(Fig.1)

Gross findings :( Fig. 1 A-C)

1- Buccal necrosisand shortening was seen in group B (G.B) pouches after the third application and disappeared in one week, resulting in reduction of the pouches' length to about $2 \mathrm{~cm}$. Comparable finding was seen in groups $(F \& G)$, i.e. lower concentrations, however their left pouches were elongated to about $2.5-3 \mathrm{~cm}$ at end of the 
experiment. Other groups $(\mathrm{C}, \mathrm{D}, \mathrm{E})$ showed delaying and milder buccal necrosis for one week, with elongated pouches to about $3.5-4 \mathrm{~cm}$, at $10^{\text {th }}$ week. (Normal pouch length is about $5 \mathrm{~cm}$ ).

2- Hair loss of the perioral area and skin lesions were extensive in G.B that were milder and delayed in the combined groups.

3- Aggregation of inflammatory cells (Fig. 2 A-C)then translocated and expelled from the sub-epithelial area upwards to outside the epithelium with normal appearing surrounding cells. These collections were seen at weeks 2-4 of all combined treatment groups. Mild sub-epithelial inflammatory infiltrate along the experimental period, only in groups $(\mathrm{F} \& \mathrm{G})$.

4- Keratin pearls (Fig. D \&E)seen as to be expelled from the epithelium, at $4^{\text {th }}$ to $6^{\text {th }}$ weeks, in all combined groups.

Histopathologic results: (H\&E stain):The pouches of the negative control (G. A)and right pouches of all experimental groups showed normal histologic appearance (Fig. 3A), with slighthyperkeratinisation of experimental groups.Due to the thin nature of the hamsters' pouch epithelial lining, evaluation of the dysplastic criteria followed El-Dakhakhny et.al.(2009)(19).

The leftpouches ofG. B, after $\mathbf{2}$ weeks of painting showed extensive necrosis of the pouches' distal part, surrounded by dense inflammatory infiltrate, a zone of granulation tissue where muscle fibers are degenerating. The rest of the lining revealed variable amounts of inflammatory infiltrate.After 4 weeks:focalareas ofmoderate dysplasia,progressed to severe dysplasia/ CIS and invasive islands confined to the papillary lesionsafter6 weeks (Fig. 3B).From the $6^{\text {th }}$ to $8^{\text {th }}$ weeks, large papillary overgrowths are seen with deeper invasion beyond these lesions. No muscle fibers could be seen at these areas, and variable inflammatory infiltrate along the pouch length. Rest of the pouch was disturbed with multiple focal thickenings, downward growths and variable degrees of dysplasia. The necrotic area was still present till end of the experiment.

G. C:After 2 weeks, moderate necrotic mass surrounded by inflammatory infiltrate then granulation tissue, where muscle fibers are degenerating, and few fibers had central nuclei (fig. 4A). The rest of the lining was devoid of inflammatory infiltrate. Focal intraepithelial collectionsof inflammatory cells along the epithelial lining. Increasing muscle fibers replacing the fat tissue (Fig.4C). After 4weeks, distal necrosis is still present, and the rest of mucosa showed focal areas of mild dysplasia with mild inflammatory infiltrate. By week 6,keratin nodules were seen with mildinflammatory infiltrate and normal muscle fibers. The rest of the epithelium showed focal hyperplasia and keratinization, with resolved necrotic end. Week 8:the epithelial lining was hyperplastic and hyperkeratinised, the lamina propriawas thicker and devoid of inflammatory infiltrate. By week 10, focal areas of mild dysplasia with normal appearing epithelium in the rest of pouch lining. The dense muscle zone was more organized with no fat tissue.

Gs. D\&E: after 2weeks, the necrotic mass was less extensive, surrounded by granulation tissue and degenerating muscle fibers. The outer zone revealed muscle fibers with multiple nuclei(Fig.4A).The rest of the epithelium and muscle fibers were of normal appearance with mild inflammatory infiltrate. After 4 weeks, the hyperplastic epithelium surrounding the necrotic mass showed mild/ moderate dysplasia (Fig.3D) the muscle fiberswere degenerating, with central nuclei of some fibers. The rest of the pouch showed focal epithelial hyperplasia, mild dysplasia, and focal aggregates of intraepithelial inflammatory cells, at different levels of the epithelial thickness. After 6-8 weeks, hyperplasia\& keratinization covering thicker fibrotic lamina propria, more muscle fibers showing multinucleation, and healed necrosis. At week 10, focal epithelial hyperplasia and keratinization. Themuscle fibers'zone was thicker and more organized, multinucleation and central nuclei were seen in some fibers, at few areasfat cells infiltrate muscle fibers.(Fig. 4B).

Gs. F\&G: after 2 weeks, the findings were comparable to G. B. However multiple areas of intraepithelial inflammatory cells' collection along the pouches' epithelial lining. Therest of the pouches were hyperplastic, and the muscle layer was cellular. From week4 to 6, focal areas of mild dysplasia were detected,muscle fibers are intermixed with fat cells. From week6 to 10:the muscle layer was of normal appearance, with central nuclei of some fibers, with thicker, fibrotic lamina propria. The epithelium showed mild/ moderate epithelial dysplasia in a focal pattern.

IHC results: Evaluation of the IHC results were subjective as mild, moderate or intense. However, nuclear localization ofNf-kB and caspase-3was consideredasactivated markers.

1-TNF- $\square$ (Fig. 5 A \&B)

a- Both pouches ofG.Aand right pouches of all experimental groups: showed mild diffuse reaction in both epithelium and muscle fibers, along the experimental period.

b- G.B: Moderate diffuse reaction of epithelial cells, inflammatory cells, endothelial cells, scattered mononuclear cells (MNC) (possibly macrophages), with mild reaction of muscle fibers. This finding was reported from the $2^{\text {nd }}$ to $8^{\text {th }}$ week, especially the tumor cells, endothelial cells and macrophages, which were moderate/ intense.

c- Left pouches of group C: from the 2 nd to 4 th weeks showed nuclear reaction of normal appearing epithelium, near area of necrosis. The intraepithelial inflammatory cells were negative, whileMNC were positive. The reaction was negative from the sixth to $10^{\text {th }} \mathrm{wks}$. 
d- Left pouches of groups D\&E: Fromthe $2^{\text {nd }}$ to $4^{\text {th }}$ weeks, nuclear reaction of epithelial cells, near the necrosis, and MNC, comparable to the combined TQ group. The reaction was negative in the epithelium and intraepithelial inflammatory cells away from the necrotic area. From weeks 6 to 10, the reaction was diffuse in the upper epithelial cells, while was positive in the blood vessels and MNC. In all cases muscle fibers showed mild diffuse reaction.

e- Left pouches of groups F\&G: from the $2^{\text {nd }}$ to $6^{\text {th }}$ weeks, it was moderate in all epithelial cells, and MNC. From the $8^{\text {th }}$ to $10^{\text {th }}$ weeks, the reaction was mild in the epithelium, moderate in MNC. Tumor cells stained moderatein the lower layers. In all cases the muscle fibers showed mild diffuse reaction.

2- Nf- $\square$ B(Figs. 6A-F)

a- G.A. and right pouches of experimental groups: Showed mild/ moderate diffuse reaction in the epithelium and moderate in muscle fibers.

b- G.B: From the 2 nd to $6^{\text {th }}$ weeks, the reaction was nuclear and cytoplasmic in all epithelial cells, inflammatory cells, blood vessels and MNC. The muscle fibers' reaction was mild. At week 8, the reaction in tumor cells wasnuclearinbasal cells, and diffuse suprabasally.

c- G.C:Atweek 2, all epithelial cells and intraepithelial inflammatory cells were positive. Degenerating muscle fibers at the necrosis area were intensely stained and moderate along the rest of the pouch. From week 4 to 8 , the reaction was mild and diffuse through the epithelial layers, blood vessels, MNC, and muscle fibers. At week 10, nuclear stain of the basal layers of rathernormal appearing epithelium. Increased bulk of the muscle zone was moderately stained.

d- Left pouches of Gs. D\&E: at week 2, there was nuclear and cytoplasmic stain of basal epithelial cells, intraepithelial inflammatory cells. At weeks 4 and 6, there was focal positive cells (nuclear) in the lower epithelial layers, of hyperplastic regions, while was diffuse in the rest of normal appearing epithelium. The thickened muscle fibers expressed mild/ moderate reaction. At week 8, focal hyperplastic areas showed positive nuclear reaction of the basal cells, diffuse cytoplasmic in the rest of epithelial lining. Byweek 10, the reaction was negative in all epithelial layers.

e- Gs. F\&G: at weeks 2 and 4, there was nuclear and cytoplasmic reaction in all epithelial layers, and the intraepithelial inflammatory cells were positive. The degenerating muscle fibers were intensely stained, while were moderate along the rest of pouches' mucosa. At week 6 there was mild/moderate diffuse reaction of suprabasal layers, with only few cells showed nuclear reaction. At week 10, few suprabasal cells expressed nuclear reaction, muscle fibers and blood vessels were mildly stained.

3-TGF- $\square$ (Figs.7A-D)

a-G.A: and right pouches of all experimental groups: the reaction was intense in all epithelial layers, muscle fibers, andMNC, while was negative in the lamina propria.

b- G.B: fromweeks2 to6,the epithelium showed mild/ moderate reaction, the degenerating muscle fibers and MNCwere mildly stained.From weeks 8 to 10 the surface epithelium and tumor cells showed intense positive reaction. Away from the necrotic area, muscle fibers showed mild /moderate stain.

c- G. C:at $2^{\text {nd }}$ and $4^{\text {th }}$ weeks, the basal epithelial cells, muscle fibers and endothelial cells stained moderate/ intense. Positive scattered MNC around the degenerating muscle fibers at the area of necrosis. At the distal end of the pouches, elongated, parallel cells showed alternating negative and positive reaction. From the $6^{\text {th }}$ to $10^{\text {th }}$ weeks,positive reaction of basal epithelial cells, thickened muscle layer with membranous reaction and MNC.

d- Gs. D\&E: at week 2, degenerating muscle fibers showed membranous stain, epithelium, muscle fibers and MNC, away from the necrosis, were intensely stained. At weeks 4 and 6, mildreaction inthe hyperplastic epithelium, muscle fibers and MNC. Fat infiltrating muscle fibers where some fibers showing central nuclei. An area with longitudinal parallel cells waspositive. At weeks 8 and 10, moderate/ intense epithelial stain, while the increased bulk of muscle fibers, endothelial cells, and MNC were mildly stained. At areas of fat infiltrating the muscle layer, some fibers showed central nuclei.

e- Gs. F\&G: from the $2^{\text {nd }}$ to $4^{\text {th }}$ weeks, mild/ moderate reaction of all epithelial layers, whilemuscle fibers and MNC were moderately stained. Near the necrosis, parallel cellular zone showed negative and positive reactions. From weeks 6 to 10, the normal appearing epithelium was moderately stained while the increased bulk of muscle fibers were mildly stained replacing fat cells.(Fig.7 C \&D).

4-Caspase3 (Fig. 8A-F)

a- G.A:and right pouches of all experimental groups: nuclear and diffuse cytoplasmic reaction in all epithelial cells. The muscle fibers and endothelial cells weremoderately stained.

b- G. B and Gs. F\&G: at weeks 2 and 4, lower basal layersof hyperplastic epithelium showed nuclear reaction near the necrotic area. The rest of epithelial lining it was diffuse and intense. At weeks 6 and 8, tumors' cells showed nuclear stain,howevermild diffuse reaction of upper layers was seen in (Gs. F\&G). The normal appearing epithelium was intensely stained. MNCbetween fat cells that is infiltrating muscle fiberswere positive. At week 
10 nuclear and mild cytoplasmic reaction of normal appearing epitheliumin Gs. F\&G, as well as the tumor cells. In all cases the muscle fibers' reaction was mild/ moderate.

c- G. C:at weeks 2 and 4,degenerating muscle fibers, at area of necrosis, were intensely stained, and the lower cells of epithelium along the rest of mucosal lining. The hyperplastic epithelium showed nuclear reaction of the lower cells. From weeks 6 to10, moderate reaction of lower epithelial cells, intense stain ofMNCbetween muscle fibers. At areas of fat infiltrating muscle fibers, some fibers showed central nuclei. In all cases the muscle fibers were mildly stained.

d- Gs. D\&E: at weeks 2 and 4, the hyperplastic epithelium around necrosis was moderately stained (nuclear and cytoplasmic), while normal appearing epithelium showed nuclear stain of basal cells. Muscle fibers showed mild/ moderate stain with central nuclei in some fibers, and MNC. From weeks 6 to 10, the reaction nuclear and cytoplasmic in all epithelial layers. The muscle layer was significantly increased with mild / moderate reaction.

\section{5-Masson's trichrome stain: (Fig.9 A-D)}

This stain was carried out to elucidate the nature of longitudinal parallel cells, thought to be regenerating muscle fibers. Green color indicates fibrous tissue and purple color indicates muscle fibers. In $G$. Conly at week 2, transition from green to purple colors were seen (Fig. 9 C \& D). While in all other groups, no distinct transition between the two colors was clear.

\section{Discussion}

This study aimed to introduce a safe and effective nanoformulation of the well known thymoquinone (TQ), as a chemo-protective and -therapeutic agent in the classic HBP/DMBA model. Along the experimental period, a promising muscle regenerative effect was noted for the used agents. TQ is well known as an anticancer phytochemical on different in vivo and in vitro models (16). In the present work liposome-encapsulated thymoquinone in nanoformulation (NTQ) was used as a chemo-protective, and -therapeutic agent, compared to bulk (TQ). The prepared NTQ at the defined size and shape (spherical, 3-25 nm) was found none toxic as determined by liver and kidney parameters (reported by a comparable study in our lab.) using the same model (15). The safety of TQ was previously confirmed, in other in vivo models, by Badary et al (1999) (30), and Nair (1991) (31). The none-toxic effect of TQ and NTQ, in the present study, was evident through the better general health performance of animals received the combined treatments compared to G. B,delaying and regressing the gross changes and the malignancy process. When the animals were left without any treatment for another 2 weeks, this protective effect was extended in the form of further regression of the clinical features and malignancy process.

Histologically, G. B showed moderate dysplasia after 4 wks, then CIS to superficial invasion after 6 wks, progressed to deeper invasion at end of the experiment. Indicating that initiating/ promoting effect of DMBA was not arrested when DMBA was stopped for 2 weeks. This might be attributed to the presence of necrotic tissue to end of the experiment. While in the groups given DMBA withlower NTQconcentrations showed mild/ moderate dysplasia after 4 wks, progressed to severe dysplasia after $6 \mathrm{wks}$, and regressed to mild/moderate dysplasia at end of the experiment, when DMBA was stopped and extended protection of NTQ. Meanwhile, Gs. C, D, E showed regression of dysplastic changes to normal appearing pouches with only mild dysplasia. This regression was reported by other researchers used TQ and PTQ in the same protocol $(2,19)$, or green tea extract, or Nigella sativa whole oil (NSO) (32, 33).

It is well documented that the main TQ activityis its anti-inflammatory effect (34), and suppression of TNF$\alpha-\mathrm{NF}-\mathrm{\kappa B}$ activation (7). That effect was on line with the intense expression of both TNF- $\alpha$ and nuclear localization of NF-кB, in the epithelium of G.B,in the present work, i.e. activating the TNF- $\alpha-\mathrm{NF}-\mathrm{kB}$ pathway. While were moderate andconfined to the basallayers of dysplastic epithelium, in early weeks (2-4 wks) of all combined groups. It is possible that the continuous DMBA painting aborted the full protective effect of TQ and NTQ, especially at early weeks of painting. In a human chronic myeloid leukemia cells (KBM-5) model, confirmed that TNF- $\alpha-\mathrm{NF}-\mathrm{KB}$ pathway was inhibited by TQ in a dose-and time-dependent manner, as well as suppressedp65 binding to DNA. Which in turn would down regulate expression of NF-кB-regulated anti-apoptotic, - proliferative, and -angiogenic gene products (4). In the present study, TNF- $\alpha$ was diffusely expressed in all epithelial, and inflammatory cells, in G. $\mathrm{B}$ and Gs. F\&G up to end of the experiment. While reduced expression of TNF- $\alpha$ and NF-KB in Gs. C, D, E, from weeks 4-10, parallel with regressed malignant transformation of the pouches' epithelium. In a cancer cell line, TQ interfered with TNF- $\alpha$ - NF-KB signaling during apoptosis-induction (7). The authors had documented its dosedependent effect, where $12.5 \mu \mathrm{m}$ had induced expression of 4 pro-apoptotic genes, $100 \mu \mathrm{m}$ induced expression of 21 genes directly involved in apoptosis, 10 genes involved in TNF signaling and 3 genes of NF-KB signaling. These results would explain the best effect of TQ and NTQ higher concentrations, where regression of dysplastic changes to near normal with only focal mild dysplastic areas, i.e. through apoptosis of mutated cells. The dose- and timedependence of TQ's anti-inflammatory effect was further revealed by healing of the necrosis after 6 wks of DMBA painting in the combined groups, partly due to expulsion of the inflammatory cells. While the necrosis and diffuse infiltration of inflammatory cells were still present to end of the experiment in group B. Matching results of a comparable study were reported when TQ loaded on gold nanoparticles (G NPs-TQ), applied topically at different 
concentrations. They were given for 2 weeks, followed by combined DMBA and all used agents for 7 and 14 weeks (3/wk). The authors found that (G NPs-TQ), when TQ concentration was $0.001 \mathrm{mg} / \mathrm{kg}$, was the most effective in regressing severe dysplasia in the DMBA-only painted pouches (showing intense nuclear NF-KB expression) to mild dysplasia and mild nuclear NF-KB expression after 7 weeks. While it had regressed the invasive carcinoma with intense nuclear NF-KB expression (DMBA-only painted pouches) to CIS and mild nuclear NF-kB expression after 14 weeks (35).

As one of the main sources of TNF- $\alpha$ is the inflammatory cells (36), in turn TNF- $\alpha$ activates NF-KB (7), extrusion of the inflammatory cells, in the present study, in all combined groups, would result in the beneficial effect of the used agents. Furthermore, these inflammatory cells' (especially groups C, D, E) wereTNF- $\alpha$ negative, while were positive for NF-kB. In contrast NF-kB was negative in the surrounding epithelial cells. A finding that confirms another direct anti-inflammatory effect of TQ and NTQ on the TNF- $\alpha$-producing cells, in turn imposing a suppressing effect on NF-KB in the epithelial cells (7), at least in early weeks of carcinogen exposure.

The extrusion of inflammatory cells and keratin pearls could be related to a previously reported finding of expulsion of what seemed dysplastic cells, in a comparable study (33) and personal communication. The author used Nigella sativa whole oil (NSO), before-, with- or after DMBA. Histologically, group of epithelial cells seen at the uppermost layer of surface epithelium, surrounded by clear zone, which was proposed to be Langerhans' cells. These findings would be another direct way of both NSO and its active ingredient TQ, to protect against the malignant process.

Another protective mechanism of TQ is activation of various apoptotic pathways in different cancer cell lines, whether through p53, (37) or caspases- (-8,-9, and-3) (29). Therefore, caspase-3 was the focus in the present in vivo model both in the epithelium and muscle fibers, due to its unexpected role in muscle regeneration (28).

The present results showed faint diffuse expression of caspase-3 in all epithelial layers and muscle fibers, in group B, along the experimental period, and early weeks of other combined treatment groups. Later weeks, it became nuclear in all combined groups. This finding indicates an apoptotic activation pathway to mutated epithelial cells by TQ and NTQ, through caspase-3. TQ and all NTQ concentrations' effect was doseand time-dependent. In G. B caspase-3 expression was mild and diffuse in all epithelial layers, i.e.DMBAsuppressed the caspase apoptotic pathway. Whereas its expression was moderate/ intensein Gs. C, D, Eparallel to the normal appearing surface epithelium, even with continuous painting of the DMBA, i.e. the agents stimulated caspase apoptotic effect.The TQ-induced caspase-3 activation was confirmed in an in vitro study, where TQ induced apoptosis through caspase-3 activation (29).

From the previous findings, it is clear that TQ and NTQ, had both protective and therapeutic effects on the HBP/DMBA model, through anti-inflammatory and apoptotic pathways, in addition of significant pouches' elongation to near normal length.

In a review of muscle regeneration, chronic inflammation and fibrosis result in interference with muscle regeneration (38), supporting the present results, where continuous DMBA painting (G. B), elicited persistent irritation ending with short pouches with no evidence of elongation till end of the experiment, even when DMBA was stopped for 2 weeks mostly due to persistence of the necrotic tissue. While in all combined groups, TQ and NTQ in a dose- and time-dependent manner hadstimulated muscle regeneration, as their pretreatment then continued with DMBAand expulsion of the inflammatory cells, had resulted in reduced tissue necrosis and faster resolution of the necrotic tissue, i.e. promoting muscle regeneration. The sequence of muscle regeneration, following its degeneration, starts in few hours to few days (38). Therefore, it was of importance to look for some important mediators of muscle regeneration as TGF- $\beta, \mathrm{TNF}-\alpha, \mathrm{NF}-\mathrm{kB}$, and caspase- 3 , where the induction of degeneration (by the carcinogen) followed by muscle differentiation (induced by the anti-inflammatory TQ and NTQ) cascade. In the present work, this combined treatment had resulted in overlap between the different cells and mediators involved in that process in favor of muscle regeneration. TNF- $\alpha$ was moderately expressed in MNC (possibly macrophages), in $\mathrm{G}$. B pouches up to end of the experiment. In parallel isNF-KB nuclear expression in the inflammatory cells, and muscle fibers. As indicated by Warren et al (2002), TNF- $\alpha$ activating NF-KB leads to proteolysis of muscle fibers (8), explaining why pouches of G. B did not show elongation. Furthermore, the authorsproposed a dual action of TNF- $\alpha$ that it activates quiescent satellite cells and enhance their proliferation and differentiation, in primary myoblasts (8). Also Li (2003) showed that TNF- $\alpha$ is mitogenic both in vitro (primary myoblasts) and in vivo through promoting activation of satellite cells in adult mouse muscles (39). In the present work, where pouches' elongation in groups C-G (no or mild inflammation and negative TNF- $\alpha$ expression), would be due to other cytokines' effect that compensate the myogenic role of TNF- $\alpha$ (40), or to a direct effect of TQ and NTQ on myogenesis. Another explanation is that low TNF- $\alpha$ concentration $(2-6 \mathrm{ng} / \mathrm{ml})$ was found to evoke TNF- $\alpha-$ receptor activation in cultured myocytes, while in higher doses $(20 \mathrm{ng} / \mathrm{ml})$ it was toxic (51) i.e. concentrationdependent. Other studies showed that repeated doses from 100 to $500 \mu \mathrm{g} / \mathrm{kg}$ of TNF- $\alpha$ had resulted in muscle protein loss or apoptosis $(24,40)$. That would explain the inability of short pouches to elongate in G.B, as the 
high inflammatory cellular infiltrate, with their expected release of higher doses of TNF- $\alpha$, leading to apoptosis of muscle fibers. Furthermore, expulsion of inflammatory cells, in the present work, following i.p. injections of TQ and all NTQ concentrations had resulted in further depletion of TNF- $\alpha$ from the area.

Philippou et al (2008) documented that after skeletal muscle injury, increased expression of TGF- $\beta$ takes place and activated in the ECM around muscle fibers (41).This note matches with the present study where infiltrated inflammatory cells in the area of tumors and around necrotic tissue were intensely expressed in groupB i.e. DMBA imposed a continuous inflammatory state that prevents myogenesis. Milder TGF- $\beta$ expression was seen in Gs. F\&G indicating their milder protective effect against the continuous DMBA painting. On the other hand, from the $2^{\text {nd }}$ to $4^{\text {th }}$ weeks in Gs. C-E, TGF- $\beta$ was expressed in the inflammatory cells around necrosis, and was membranous in muscle fibers. The stain was fading from the $8^{\text {th }}$ to $10^{\text {th }}$ weeks, where bulk muscle zone had been formed, i.e. groups C-E had suppressed TGF- $\beta$ activity even with continuous DMBA painting, in favor of evident myogenic effect. This further confirms a dose- and time-dependent effect of the used agents on the myogenic process.

It was found that TGF- $\beta$ can cause satellite cell apoptosis so potently inhibit their proliferation and fusion (42). This could explain the present findings in G.B and early weeks of combined treatments, fat cells infiltrated muscle fibers, and at areas thickened fibrotic lamina propria (at the expense of the muscle layer) was clear, in parallel with expression of TGF- $\beta$.

A zone of parallel elongated muscle fiber-like cells were seen atthe pouches' distal part,at early weeks, which by Masson's trichrome stain, showed a transition from fibrotic (green color) to myogenic tissue (purple color) in group $\mathrm{C}$, while was indistinct in other groups. This indicates a superior TQ myogenic effect over other combined NTQ- groups,

As TGF- $\beta$ is released by infiltrating monocytes, local parenchymal cells, myo-fibroblasts, cells of the extracellular matrix, and epithelial cell (43), it was expressed in the scattered MNC, muscle fibers, epithelial cells, and endothelial cells, in the present work. This expression was in early weeks (2-4 wks) of all combined groups. The clinical pouches' elongation with fading of TGF- $\beta$ expression in the muscle fibers up to end of the experiment, may explain its dual effect on myogenesis. Monocytes, supposed to be macrophages, were TGF- $\beta$ positive with some muscle fibers showing central nucleation (a sign of muscle regeneration) (44), even in areas of fat infiltrating muscle layer.

Along the experimental period, the MNC between muscle fibers, were TNF- $\alpha$ and TGF- $\beta$ positive, where these MNC are of their major sources $(8,45)$. The role of macrophages in muscle regeneration was documented by their early presence (pro-inflammatory phenotype or the M1) with neutrophils to phagocytose necrotic tissues and degenerating muscle fibers. Two days later, M2 cells (the anti-inflammatory phenotype) appear to activate satellite cells, followed by muscle regeneration in 4-10 days and muscle maturation from 1015 days (44, 46, and 47). In the present study, TNF- $\alpha$-positiveMNC, between muscle fibers, were seen in the first 4wks of groups C, D, Eespecially at areas of fat cell's infiltration between muscle fibers. These positive cells had disappeared in the following weeks to end of the experiment, where the pouches were elongated clinically. On the other hand they were positive in groups B, F, G up to end of the experiment. It is possible that they could be persistent M1 macrophages that prevented regeneration with continuous DMBA painting and the less protective effect of lowest NTQ concentrations. In these pouches, many areas showed fat replacing muscle fibers.

Another finding is the membranous localization of TGF- $\beta$ of degenerating muscle fibers in early weeks of combined treatments (2-4 weeks). This localized stain could be the PDGF+-cells which differentiate to stimulate fat tissue formation and/ or fibrosis. These cells are distinct from satellite cells (which were subjected to apoptosis under the effect of TGF- $\beta$ ) (48). Philippou et al (2008) showed that TGF- $\beta$ was expressed, after muscle injury, around muscle fibers (at the ECM) (41). Meanwhile, Li et al (2004) results showed that TGF- $\beta$ elevated level in the area of muscle injury was not only due to infiltrating lymphocytes but to the local myogenic cells (42). A finding that explains its membranous expression where no inflammatory cells were found in these combined treatment groups, in the present work. Also it explains the dense lamina propria (fibrosis) at these pouches due to the known TGF- $\beta$ effect on collagen formation and inhibition of matrix degradation (45). Its dual effect (from the present work) appears in its positive expression in the epithelium and degenerating muscle fibers in G. B and early weeks of all combined groups, i.e. pro-apoptotic effect in favor of fibrosis. While, its pro-myogenic effect was evident in its expression at the distal portions of elongating muscle fibers (by end of the experiment), with some fibers showing central nuclei and multinucleated fibers.

Of interest was the contribution of caspase-3 to differentiation of various cells including muscle cells (49), which was explained as that differentiating myoblasts share a remarkable similarity with many key cellular alterations common for apoptosis (28). The role of caspase-3 was explained by Fernando et. al. (2002) where skeletal muscle differentiation depends on caspase- 3 activity. They showed that inhibition of caspase-3 activity leads to dramatic reduction in both myotubes/ myofibers' formation. Their results confirmed a unique role for a caspase-3-mediated signal cascade in promoting muscle regeneration (28). The present results confirmed the myogenic effect of caspase- 
3, where mild expression of caspase-3 in groups B, F, G along the experimental period, in parallel with non-elongated, or slightly elongated pouches, respectively. Whereas, its expression was moderate in muscle fibers showing increased muscle bulk along the experimental period, in groups C, D, E. Same expression level, in these groups, was found in areas of fat infiltrating muscle fibers, with multinuclei or central nuclei in some fibers, i.e. intermixed degenerating/ regenerating effect of the combined groups, in favor of muscle regeneration. A study had explained the effect of caspase-3, that initiation of myogenesis is required to remove a population of myoblasts (through apoptosis) which would normally inhibit the differentiation process. Alternatively, increased caspase-3 expression may lead to intracellular alterations, in turn activate a differentiation process (28). This effect was clear, in the present results, in early weeks of combined treatment groups, where the evidence of new muscle formation (central nuclei and multinucleated fibers)(49), near the necrotic mass was seen, i.e. dedifferentiation of degenerating cells to regenerate new cells. This is revealed by positive caspase- 3 of degenerating muscle fibers with regeneration potential of these cells. In an in vitro study showed that not all myoblasts revealed morphologic signs of apoptosis (caspase- 3 positive cells), but rather related to myogenic differentiation. The peak of highest expression of caspase-3 (24 hours), followed by its decrease to its base level in 48 hours, the time of maximum differentiation and myotubes' formation had been reached(50). These observations, of close timing, were not followed in the present in vivo work, which aimed to evaluate the carcinogenesis process, not muscle regeneration effect. Also the systemic route of TQ\& NTQ, would make the timing is longer than 2 daysseen in tissue culture. However, their pretreatment would offer enhanced and extended effect than if given with-, or after DMBA painting. Although, the classic HCP/DMBA model for oral cancer-induction was used for decades to evaluate the effect of many chemo-preventing and -therapeutic agents, the present study records new findings that need in-depth studies for explanation and evaluation. For example: the expulsion of keratin nodules from the surface epithelium, the aggregation and expulsion of inflammatory cells in the combined groups, surrounded by normal appearing epithelial cells, and finally, the muscle regenerative effect. These findings shed light on the differentiating potential of TQ and its nano-formulation on keratinocytes, fibroblasts and muscle fibers.

\section{Conclusion}

This work showed that it is possible to induce muscle regeneration in a toxic and continuous inflammatory/ mutagenic state. Although higher concentrations of NTQ showed comparable beneficial effects to TQ on the carcinogenesis process and myogenic potentiality, NTQ will be more economical with reducing the required dose for each disease.

\section{Recommendations}

Using higher doses / concentrations for both TQ and NTQ, with an in-depth molecular evaluation of the presented results in higher models, as well as other pathologic conditions.

NOTE: A more concise, closer time interval follow-up of initiated muscle regeneration is going on in our lab.

\section{Acknowledgement}

A sincere thanks to Dr. Mona Bakr, and Dr. Ola Al-Borady (Nano-Tech., $6^{\text {th }}$ October, Egypt), for kindly preparing, characterization and supplying the NTQ in its required concentrations. Our deepest thanks toour fellows Randa El-Sherbiny, and Sara El-Khoriby (Associate Lecturers), for their sincere help. The authors declare that they have noconflicts of interest.

\section{References}

[1]. Bedi P, Priyanka S. Effects of garlic against 7-12, dimethylbenza (a) anthracene-induced toxicity in Wistar albino rats. Asian J Pharmac Clin Res. 2012; 5(4): 170-3.

[2]. Hassan MA, El-Dakhakhny M. Effect of some Nigella sativa constituents on chemical carcinogenesis in hamster cheek pouch. J Egy Soci Pharmac Exper Ther. 1992; 11: 675-91.

[3]. Schneider-Stock R, Fakhoury IH, Zaki AM, El-Baba CO, Gali-Muhtasib HU. Thymoquinone: fifty years of success in the battle against cancer models. Drug Disc Today. 2014; 19(1): 18-30.

[4]. Sethi G, Ahn KS, Aggarwal BB. Targeting nuclear factor-K B activation pathway by thymoquinone: role in suppression of antiapoptotic gene products and enhancement of apoptosis. Mol Ca Res. 2008; 6 (6): 1059-70.

[5]. Rahmani AH, Alzohairy MA, Khan MA, Aly SM. Therapeutic implications of black seed and its constituent thymoquinone in the prevention of cancer through inactivation and activation of molecular pathways. Evidence-Based Complem Altern Med.2014; 2014: $1-13$.

[6]. Guida MS, El-Aal AA, Kafafy Y, Salama SF, Badr BM, Badr G. Thymoquinone rescues T lymphocytes from gamma irradiationinduced apoptosis and exhaustion by modulating pro-inflammatory cytokine levels and PD-1, Bax, and Bcl-2 signaling. Cell Physiol Biochem. 2016; 38:786-800.

[7]. Sakalar C, Yuruk M, Kaya T, Aytekin M, Kuk S, Kanatan H. Pronounced transcriptional regulation of apoptotic and TNF-Nfkappa-B signaling genes during the course of thymoquinone mediated apoptosis in HeLa cells. Mol Cell Biochem. 2013; 383:24351.

[8]. Warren GL, Hulderman T, Jensen N, McKinstry M, Mishra M, Luster MI, et al. Physiological role of tumor necrosis factor alpha in traumatic muscle injury. FASEB J. 2002; 16: 1630-2.

[9]. Lemos D, Babaeijandaghi F, Low M, Chang C-K, Lee ST, Fiore D, et al. Nilotinib reduces muscle fibrosis in chronic muscle injury by promoting TNF-mediated apoptosis of fibro/adipogenic progenitors. Nature Med. 2015; 21(7): 788-94.

[10]. El-Sayed I H. Huang X, El-Sayed MA. Surface plasmon resonance scattering and absorption of anti-EGFR antibody conjugated gold nanoparticles in cancer diagnostics: applications in oral cancer. Nano Lett. 2005; 5 (5): 829-34. 
[11]. Siddiqui IA, Adhami VM, Ahmad N, Mukhtar H. Nanochemoprevention: sustained release of bioactive food components for cancer prevention. Nutr Ca. 2010; 62: 883-90.

[12]. Augustin MA, Sanguansri-L, Lockett T. Nano- and micro-encapsulated systems for enhancing the delivery of resveratrol. Ann NY Acad Sci. 2013; 1290(1): 107-12.

[13]. Bai T, Lian LH, Wu YL, Wan Y, Nan JX. Thymoquinone attenuates liver fibrosis via P13K and TLR4 signaling pathways in activated hepatic satellite cells. Int Immunopharm. 2013; 15:275-81.

[14]. Mahmoud AM, Ahmed OM, Galaly SR, Thymoquinone and curcumin attenuate gentamicin- induced renal oxidative stress, inflammation and apoptosis in rats. EXCLI J. 2014; 13: 98-110.

[15]. El-Khoriby SAS. Chemopreventive effect of different doses of nano-thymoquinone on chemically-induced oral carcinogenesis. M.Sc. thesis, Suez Canal University, Egypt, 2015.

[16]. Singh A, Ahmad I, Akhtar S, Jain GK, Iqbal Z, Talegaonkar S, et al. Nanocarrier-based formulation of thymoquinone improves oral delivery: Stability assessment, in vivo and in vitro studies. Colloids \& Surfaces B: Biointerfaces. 2013, 102; 822-32.

[17]. Odeh F, Ismail SI, Abu-Dahab R, Mahmoud IS, Al-Bawab A. Thymoquinone in liposomes: a study of loading efficiency and biological activity towards breast cancer. Drug Deliv. 2012, 19(8): 371-7.

[18]. El-Sherbiny RHM. Effect of different nano-thymoquinone concentrations on the chemically-induced epithelial dysplasia in the hamster buccal pouch. M.Sc. thesis, Suez Canal University, Egypt, 2016.

[19]. El-Dakhakhny M, Hassan MA, Abdel-Aziz G. Effect of thymoquinone and polythymoquinone on chemically-induced oral dysplasia. (Experimental study). (Part 1). Int J Acad Res. 2009; 1(2): 107-17.

[20]. Chargé SBP, Rudnicki MA. Cellular and molecular regulation of muscle regeneration. Physiol Rev. 2004; 84: 209-38.

[21]. Ciciliot S, Schiaffino S. Regeneration of mammalian skeletal muscle. Basic mechanisms and clinical implications. Curr Pharm Des. 2010; 16: 906-14.

[22]. Lefaucheur JP, Sèbille A. Muscle regeneration following injury can be modified in vivo by immune neutralization of basic fibroblast growth factor, transforming growth factor beta 1 or insulin-like growth factor 1. J Neuroimmunol. 1995; 57: 85-91.

[23]. Cohn RD, van Erp1 C, Habashi JP, Solimani AA, Klein EC, Lisi MT, et al. Angiotensin II type I receptor blockade attenuates TGF-beta-induced failure of muscle regeneration in multiple myopathy states. Nat Med. 2007; 13: 204-10.

[24]. Collins RA, Grounds MD. The role of tumor necrosis factor-alpha (TNF- $\alpha$ ) in skeletal muscle regeneration: studies in TNF- $\alpha$ (-/-) and TNF- $\alpha(-/-) /$ LT- $\alpha$ (-/) mice. J Histochem Cytochem. 2001; 49(8): 989-1001.

[25]. Shoieb AM, Elgayyar M, Dudrick PS, Bell IL, Tithof PK. In vitro inhibition of growth and induction of apoptosis in cancer cell lines by thymoquinone. Int J Oncol. 2003; 22:107-13.

[26]. Paramasivam A, Sambantham S, Shabnam J, Raghundakumar S, Anandan B, Rajiv R, et al. Anti-cancer effects of thymoquinone in mouse neuroblastoma (Neuro-2a) cells through caspase-3 activation with down-regulation of XIAP. Tox Lett. 2012 ; 213 (2, 3): 1519.

[27]. Janicke RU, Sprengart MI, Wati MR, Porter AG. Caspase-3 is required for DNA fragmentation and morphological changes associated with apoptosis. J Biol Chem. 1998; 273: 9357-60.

[28]. Fernando P, Kelly JF, Balazsi K, Slack RS, Megeney LA. Caspase 3 activity is required for skeletal muscle differentiation. PNAS. 2002; 99(17):11025-30.

[29]. El-Mahdy MA, Zhu Q, Wani G, Wani AA. Thymoquinone induces apoptosis through activation of caspase-8 and mitochondrial events in p53-null myoblastic leukemia HL-60 cells. Int J Cancer. 2005; 117: 409-17.

[30]. Badary OA, Al-Shabanah OA, Nagi MN, Al-Rikabi AC, Elmazar MM. Inhibition of banzo(a)pyrene-induced forestomach carcinogenesis in mice by thymoquinone. Eur J Cancer Prev. 1999; 8: 435-40.

[31]. Nair SC, Salomi MJ, Panikkar B, Panikkar KR. Modulatory effects of Crocus sativus and Nigella sativa extracts on cisplatininduced toxicity in mice. J Ethnopharm. 1991; 31: 75-83.

[32]. Abdel-Aziz DM. Chemopreventive effect of green tea on 9, 12 dimethylbenza-(a)-anthracene (DMBA)-induced hamster buccal pouch carcinogenesis. M.Sc. thesis Suez Canal University, Ismailia, EGYPT, 2008.

[33]. Hassan, M. Nigella sativa extract and dysplasia-chemically-induced in the hamster cheek pouch epithelium. Proc Am Acad Oral Path. 1985; 39: 19(A).

[34]. Woo CC, Kumar AP, Sethi G, Tan KHB. Thymoquinone: Potential cure for inflammatory disorders and cancer. Bioch Pharm. 2012; 83: 443-51.

[35]. Hassan MH, Abou El-Nour KM, El-Azab MF, Shata MS. Nano-chemoprevention of oral squamous cell carcinoma using thymoquinone loaded on gold nanoparticles. Proc 3rd Inter Conf on Nanotechnology and its applications (ICNA-III-2016), (22nd 25th Feb., 2016), Hurghada, EGYPT.

[36]. Tracey KJ, Cerami A. The biology of cachectin/tumor necrosis factor. In: Habenicht A. ed. Growth Factors, Differentiation factors, and Cytokines. Berlin, Sprin-Verlag, p356, 1990.

[37]. Gali-Muhtasib H, Diab-Assaf M, Boltze C, Al-Hmaira J, Hartig R, Roessner A, et al. Thymoquinone extracted from black seed triggers apoptotic cell death in human colorectal cancer cells via a p53-dependent mechanism. Int J Oncol. 2004a; 25: 857-66.

[38]. Musarò A. The basis of muscle regeneration. (Review article). Adv Biol. 2014; 2014:1-16.

[39]. Li YP. TNF-alpha is a mitogen in skeletal muscle. Am J Physiol Cell Physiol. 2003; 285: C370-C6.

[40]. Hodge-Dufour J, Marino MW, Horton MR, Jungbluth A, Burdick MD, Strieter RM, et al. Inhibition of interferon gamma-induced interleukin-12 production: a potential mechanism for the anti-inflammatory activities of tumor necrosis factor. Proc Natl Acad Sci USA. 1998; 95: 13806-18.

[41]. Philippou A, Maridaki M, Koutsilieris M. The role of urokinase-type plasminogen activator (uPA) and transforming growth factor beta 1 (TGF beta 1) in muscle regeneration. In Vivo 2008; 22: 735-50.

[42]. Li T, Foster W, Deasy BM, Chan Y, Prisk V, Tang Y, et al. Transforming growth factor- $\beta 1$ induces the differentiation of myogenic cells into fibrotic cells in injured skeletal muscle: a key event in muscle fibrogenesis. Am J Path. 2004; 164(3): 1007-19.

[43]. Mendias C L, Gumucio JP, Davis ME, Bromley CW, Davis CS, Brooks SV. Transforming growth factor-beta induces skeletal muscle atrophy and fibrosis through the induction of atrogin-1 and scleraxis. Muscle\& Nerve. Jan 2012 (wilyonlinelibrary.com), DO1 10.1002/mus.22232.

[44]. Mounier R, Theret M, Arnold L, Cuvellier S, Bultot L, Göransson O, et al. AMPK- $\alpha 1$ regulates macrophage skewing at time of resolution of inflammation during skeletal muscle regeneration. Cell Metab. 2013; 18: 251-64.

[45]. 45-Border WA, Noble NA. Transforming growth factor- $\beta$ in tissue fibrosis. N Engl J Med. 1994; 10: $1286-92$.

[46]. St Pierre BA, Tidball JG. Differential response of macrophage sub-populations to soleus muscle reloading after rat hindlimb suspension. J Appl Physiol. 1994; 77(1): 290-7.

[47]. Arnold L, Henry A, Poron F, Baba-Amer Y, van Rooijen N, Plonquet A, et al. Inflammatory monocytes recruited after skeletal muscle injury switch into anti-inflammatory macrophages to support myogenesis. J Exper Med. 2007; 204(5): 1057-69. 
[48]. Uezumi A, Fukada S, Yamamoto N, Takeda S, Tsuchida K. Mesenchymal progenitors distinct from satellite cells contribute to ectopic fat cell formation in skeletal muscle. Nature Cell Biol. 2010; 12: 143-53.

[49]. Olguin HC. Regulation of Pax 7 protein levels by caspase-3 and proteosome activity in differentiating myoblasts. Biol Res. 2011; 44: 323-7.

[50]. Hunt LC, Upadhyay A, Tazayeri JA, Tudor EM, White JD. Caspase-3, myogenic transcription factors and cell cycle inhibitors are regulated by leukemia inhibitory factors to mediate inhibition of myogenic differentiation. Skeletal Musc J. 2011; 1: 1-13. Tables and Figures Legends

[51]. Table (1): Showing a summary of different groups, their treatment protocol, and main histopathologic findings.

Figure (1): (A-C): Gross appearance of the hamsters' pouches: A: Normal appearing left pouch from the combined TQ group at week 10, note: no evidence of inflammation or any gross lesions. B: group B painted pouch at end of the experiment, note inflamed surface with multiple lesions and necrotic tissue (arrow). C: Left pouch from groups $(F \& G)$ at weeks $4-6$ showing remains of necrotic tissue is expelling out (arrow).
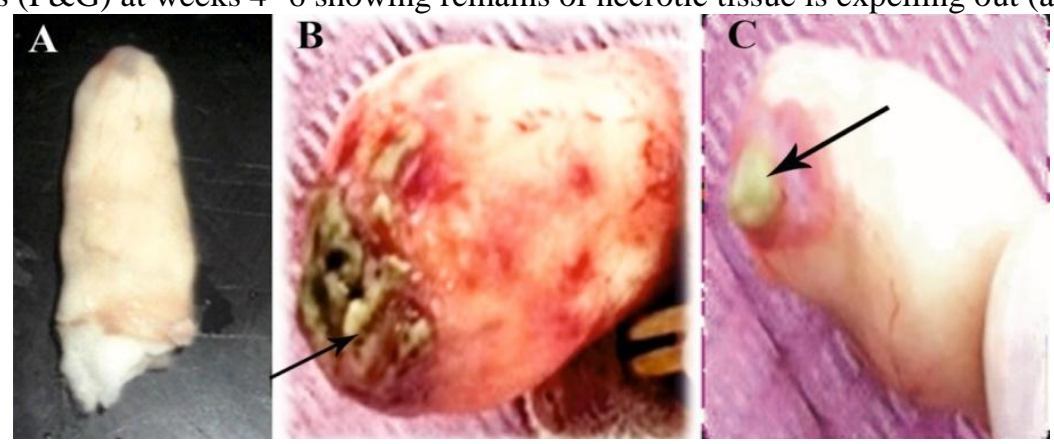

Figure (2): (A-C): Sections from combined groups showing sub-epithelial collection of inflammatory cells, and their sequential expulsion from the surface (weeks 2 -4) (H\&E x20). (D and E): Sections from groups C, D\&E at weeks 4-6, showing keratin nodules as to be expelled from the surface (H\&E x20). (F): Section from a previous study (33), where NSO was used in the HBP/DMBA model. Note: epithelial cells extruding from the epithelium surrounded by clear zone (arrow) (H\&E. x40).
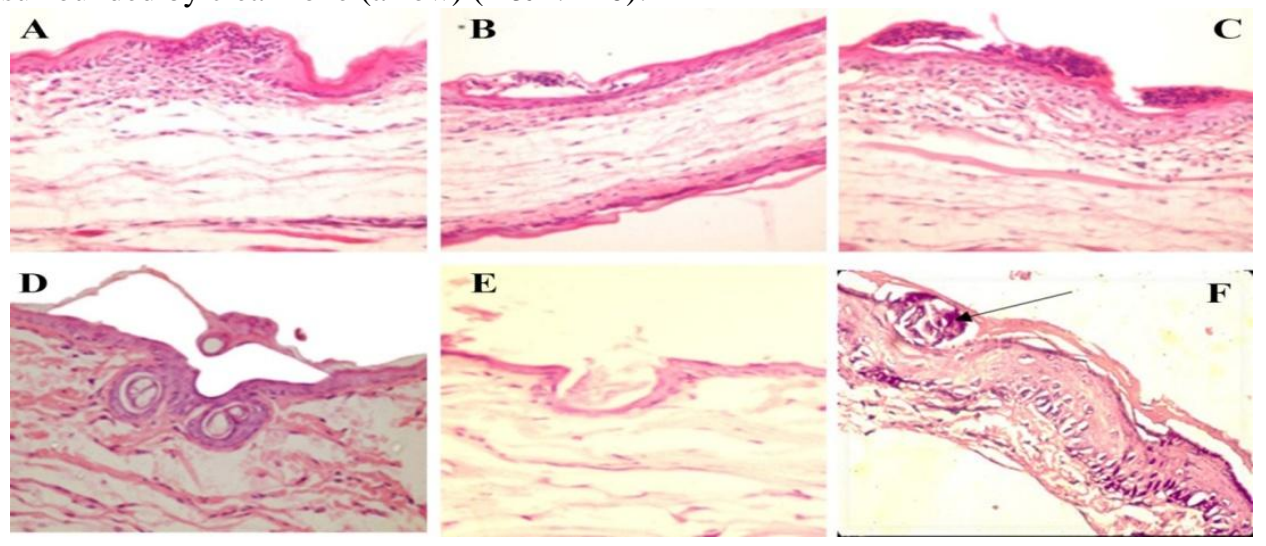

Figure (3): A: Section from group A (H\&E x40). B: Section from G.B atweek6, showing severe dysplasia, increased inflammatory infiltrate and superficial invasion of tumor cells (H\&E 10). C: Section from G.B at end of the experiment showing larger exophytic nodule and deeper invasion (H\&E x10). D: Section from a combined group at week 4 showing mild/moderate dysplasia, near the necrotic tissue (H\&E x 40).
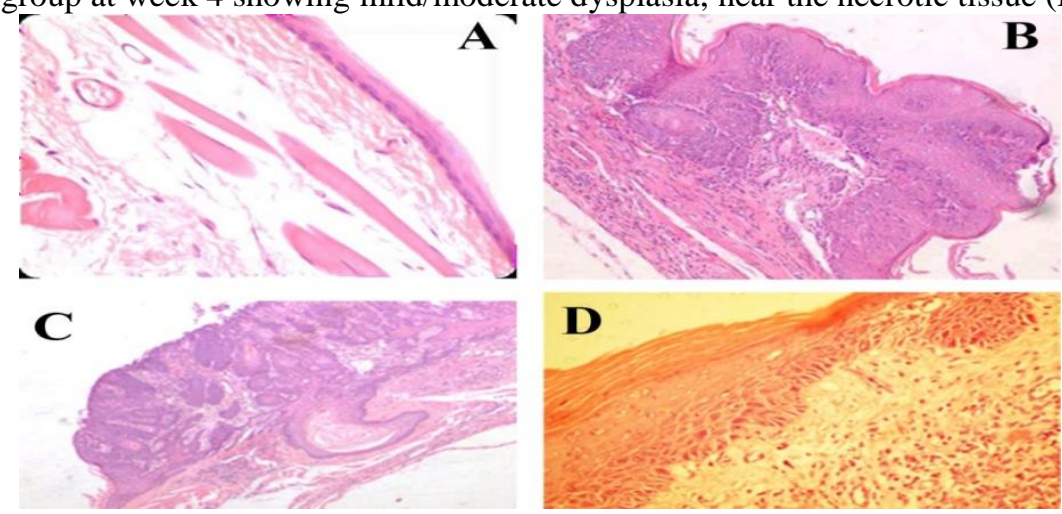
Figure(4): A: Section from groups C-G showing degenerating muscle fibers with multinuclei of some fibers (arrows) at the area of necrosis, (H\&E x40). B: Section from group D at week 6, showing intermixed fat and muscle fibersin an area of normal looking epithelium (H\&E x10). C: Section from the same group at weeks 8-10, showing increase in muscle fiberswith hyperplastic/ hyperkeratinised epithelial surface (H\&E x20).
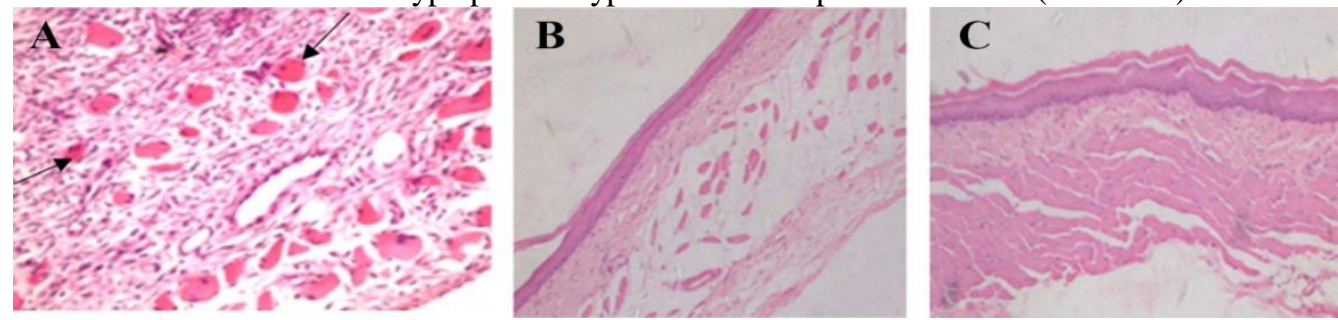

Figure (5): A-Section from G.B at week 8, showing positive nuclear reaction for TNF- $\alpha$ at the lower layers of tumor nests (TNF- $\alpha$ x20). B: Same group at the rest of mucosa (where no tumors), showing positive nuclear and cytoplasmic reaction in the lower layers (TNF- $\alpha$ x40)
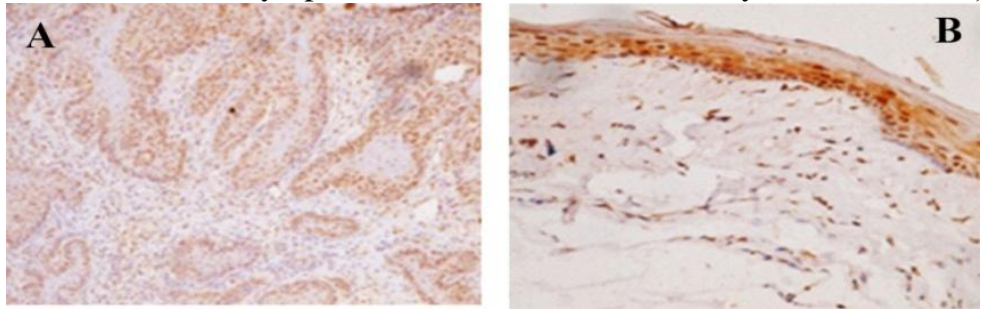

Figure (6): A: Section from G.B at week 8, showing tumor mass with activated NF-kB of basal and suprabasal cells (NF$\mathrm{kB}$ x40).B: Section from G. D moderate reaction in a thicker muscle layer (NF-kB x20). C: Section from group D at week 2, all epithelial cells were intensely stained (nuclear) in normal appearing epithelium, while was moderate and diffuse in the muscle fibers infiltrated by fat tissue (NF-kB-x20). D: Section from groups D\&E after 2 weeks, showing extrusion of inflammatory cells (arrow) from the epithelium which are positive for NF-kB, and lower epithelial cells showed nuclear reaction (thick arrow) (NF-kB x 40). E: Section from the same groups at week 4, milder nuclear reaction in hyperplastic epithelium covering increasing muscle fibers and positive MNC between mildly stained muscle fibers away from the necrosis (arrows) (NF-kB x20). F: Same groups at week 10, showing nuclear stain of basal cells only and moderate diffuse reaction of increased muscle bulk (NF-kB-x20).
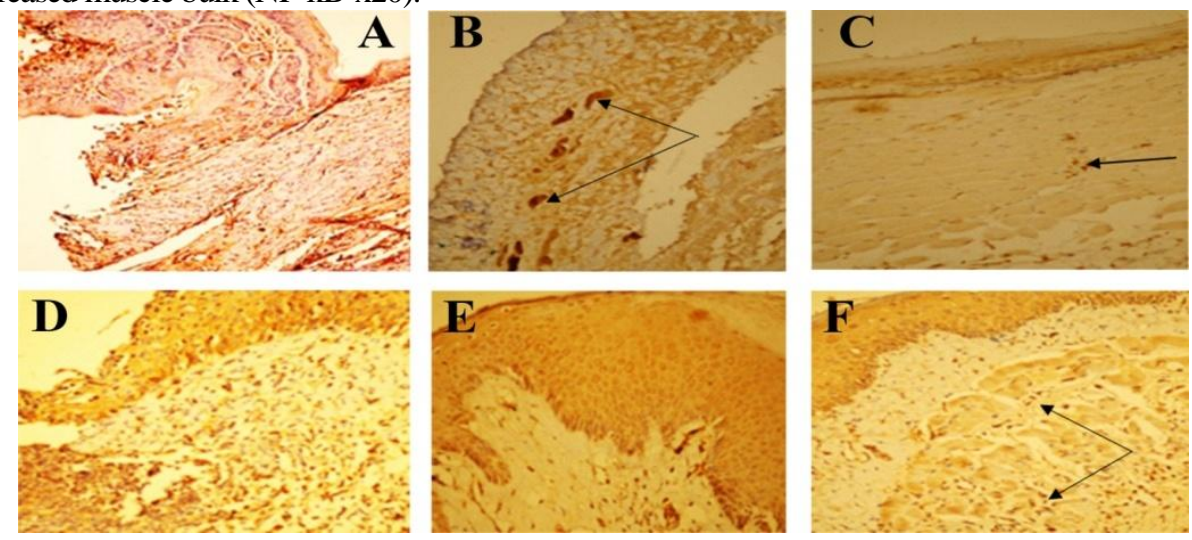

Figure (7): A: Section from Gs.D\&E at weeks 2-4, showing mild epithelial reaction with expulsion of inflammatory cells (intensely stained). Note: intermixed fat cells and intense stain of degenerating muscle fibers (TGF- $\beta$ x20). B: Section from same groups at weeks 4-6, negative/mild epithelial reaction, mild/moderate muscle fibers' reaction of increased muscle fibersreplacing fat tissue (TGF- $\beta$ x10). C: Section from same groups at week 6 showing negative reaction of normal appearing epithelium, and increasing muscle fibers (TGF- $\beta$ x20). D: Same groups at week 10, showing moderate/intense stain of normal appearing epithelium covering mildly stained bulk muscle tissue (TGF- $\beta$ $\mathrm{x} 10)$. 

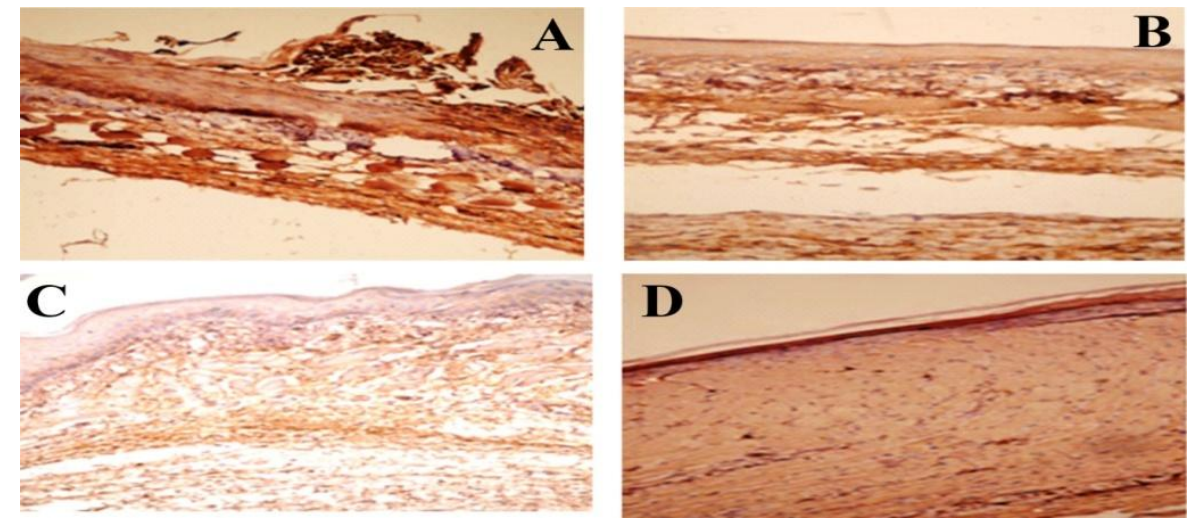

Figure (8): A: Section from G.B at week 8, showing a tumor near the necrotic mass, with dense fibrotic lamina propria. Positive basal tumor cells only (Caspase-3 x20). B: Section from G.C at week 4 around the necrotic mass, showing intense stain of degenerating muscle fibers (arrows) (Caspase-3-x10). C: Section from same group at week 10 showing increased bulk of muscle layer and positive MNC (thick arrow) Note:epithelial nuclear reaction of lower layers (Caspase-3-x20). D-F: Sections from Gs. C-E at week 4, D: group C showing hyperplastic epithelium around the necrosis that was moderately stained, and cells of granulation tissue. E: Same groups at week 6, showing epithelial hyperplasia and nuclear/cytoplasmic reaction.F:Same groups at week 10, showing moderate nuclear/cytoplasmic epithelial reaction and positive MNC (arrows) between moderately stained increased muscle fibers (Caspase-3 x40).
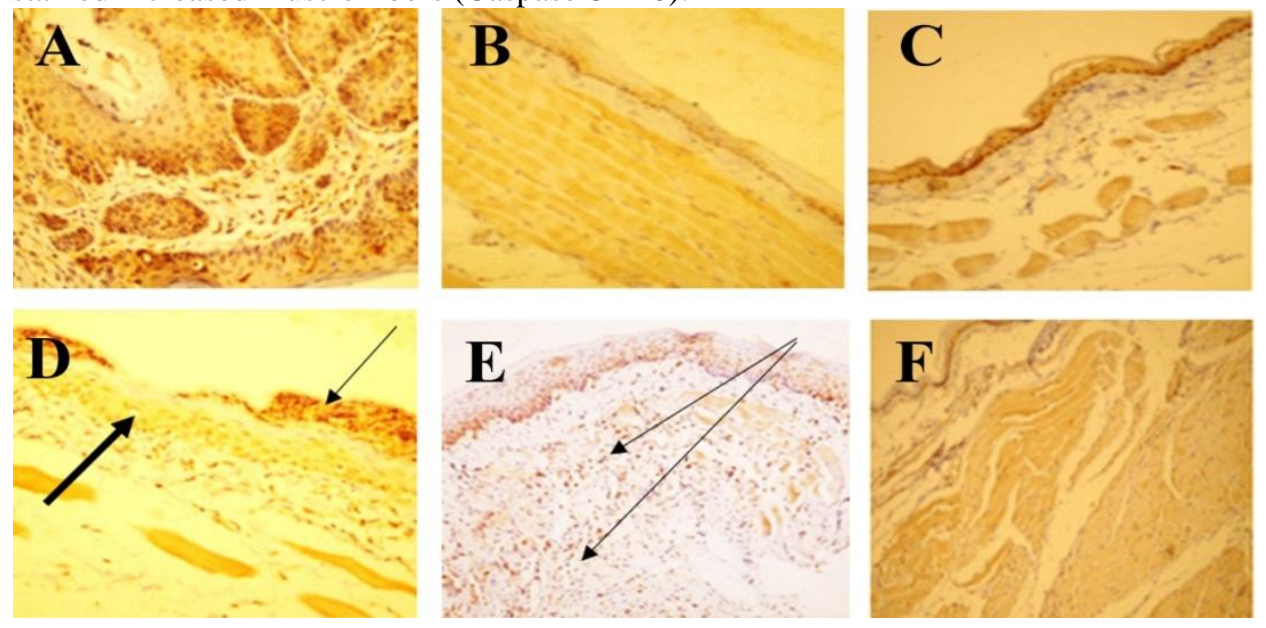

Figure (9): A: Section from combined groups at week 8, showing the distinct three layers (epithelium, lamina propria, and muscle fibers). B: Section from the combined groups, showing intermixed stain of fibrotic tissue within muscle fibers (arrows) (Masson's trichrome stain x10).C: Section from group C at week 2 showing transition from fibrotic (green) to muscular tissue (purple) \{arrows\} (Masson's trichrome x20). D: Higher power. (Masson's trichrome x40).
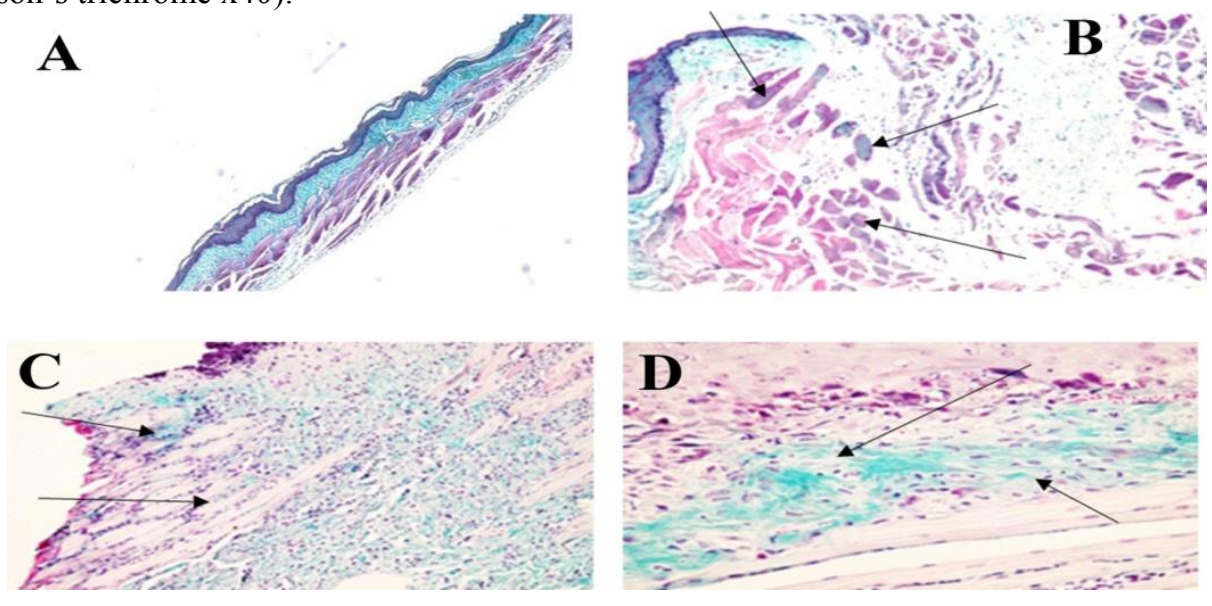
Table 1: Showing a summary of different groups, their treatment protocol, and main histopathologic findings.

\begin{tabular}{|c|c|}
\hline Group and treatment (s) & Main histopathologic finding(s) \\
\hline A- $\quad$ Negative control group (25 hamsters) & Normal pouch mucosa \\
\hline $\begin{array}{ll}\text { D- } & \text { NTQ } 0.1 \mathrm{mg} / \mathrm{kg} \text { (pretreatment), followed by combined } \\
\text { treatment ( } 25 \text { hamsters })\end{array}$ & From moderate to focal mild dysplasia \\
\hline $\begin{array}{ll}\text { F- } & \text { NTQ } 0.025 \mathrm{mg} / \mathrm{kg} \text { (pretreatment), followed by combined } \\
\text { treatment ( } 25 \text { hamsters) }\end{array}$ & $\begin{array}{l}\text { From moderate to severe dysplasia, regressed to focal mild/ } \\
\text { moderate dysplasia }\end{array}$ \\
\hline $\begin{array}{ll}\text { G- } & \text { NTQ } 0.01 \mathrm{mg} / \mathrm{kg} \text { (pretreatment), followed by combined } \\
\text { treatment ( } 25 \text { hamsters })\end{array}$ & $\begin{array}{l}\text { From moderate to severe dysplasia, regressed to focal mild/ } \\
\text { moderate dysplasia }\end{array}$ \\
\hline
\end{tabular}

